\title{
Issues in the Design of Studies for the Economic Evaluation of New Atypical Antipsychotics: the ESTO Study
}

\author{
M.F. Drummond ${ }^{1}$, M.R.J. Knapp ${ }^{2}$, T.P. Burns ${ }^{3}$, K.D. Miller ${ }^{4}$ and P. Shadwell ${ }^{4}$ \\ ${ }^{1}$ Centre for Health Economics, University of York, York YO1 5DD, UK \\ ${ }^{2}$ Centre for the Economics of Mental Health, 7 Windsor Walk, Denmark Hill, London SE5 8BB, UK \\ ${ }^{3}$ St George's Medical School, Section of Community Psychiatry, Cranmer Terrace, London SW17 ORE, UK \\ ${ }^{4}$ Zeneca Pharmaceuticals, Alderley House, Alderley Park, Macclesfield SK10 4TF, UK
}

\begin{abstract}
Background: Increasing attention is being focused on the costs of healthcare and the need for cost-effective treatments. Drugs for schizophrenia have not escaped this scrutiny, especially now that several new agents are available, with acquisition costs substantially higher than for established therapies. However, most of the existing evaluations of new drugs for schizophrenia have weak designs, either comparing health care costs before and after introduction of the new drug, or being based on modelling approaches incorporating numerous assumptions.
\end{abstract}

Aim of the Study: The aim of the study was to discuss and resolve the key design issues in the planning of a prospective randomized trial to assess the socio-economic impact of a new atypical antipsychotic (quetiapine).

Methods: Key methodological issues were identified and discussed in the context of the economic evaluation being planned. These were patient recruitment and entry criteria, selection of comparator drug, blinding of doctor and patient, range of socio-economic outcomes, length of follow-up and sample size.

Results: The resulting economic evaluation, the ESTO study, was an international multi-centre randomized controlled trial, with concurrent data collection for a wide range of clinical, economic and quality of life outcomes. The trial had a pragmatic design, enrolling patients experiencing an acute exacerbation on existing therapy. In addition to the presenting exacerbation, patients must have had at least one hospitalization or documented evidence of exacerbation within the previous three years. On admission to the study, existing psychotic medication was withdrawn prior to randomization to quetiapine or haloperidol. Doses of both drugs were titrated up to an optional dose, with flexibility for additional increases if required.

Both patients and doctors were blinded to treatment allocations, on the grounds that, since quetiapine was still in development, unblinded assessments of efficacy would not be credible. Patients were followed for 1 year, irrespective of whether they withdrew from study medication.

A wide range of socio-economic outcomes was assessed, including costs falling on the healthcare sector, other agencies and the family. In addition data were collected on patients' earnings and quality of life, measured by the Short-Form 36 health profile. Data were also collected on a range of clinical measures, such as

\footnotetext{
* Correspondence to: Professor M F Drummond, Centre for Health Economics, University of York, Heslington, YORK, YO1 5DD.

Funding: Zeneca Pharmaceuticals.
}

the Positive and Negative Syndrome Scale (PANSS), the Clinical Global Impressions (CGI), the AIMS neurological rating scale and the neurological rating scale of Simpson and Angus. This was to assess whether changes in socio-economic end points were indeed matched by changes in the patient's clinical condition.

Conclusions: The design of studies such as ESTO is inevitably a compromise between control and pragmatism. For example, whilst blinding of doctor and patient may reduce potential bias, this may cause difficulty with compliance owing to the use of additional dummy medications. Despite these compromises, the ESTO study should provide a more reliable assessment of the socio-economic outcomes of a new anti-psychotic and has attracted the widespread support of analysts and investigators. It has already served as a template for other studies and, if the methodology is successful, will have implications for the assessment of similar drugs in the future. (C) 1998 John Wiley \& Sons, Ltd.

Received 18 July 1997; accepted 11 December 1997.

\section{Introduction}

Schizophrenia is a common serious mental illness which imposes a burden not only on the patient, but also on carers, hospitals, and society. Furthermore, it is a relatively expensive disease to treat. The direct cost of treating schizophrenia in the UK has been estimated at $£ 310$ million, which is similar to that for asthma (approximately $£ 340$ million), and slightly less than that of major depression ( $£ 417$ million). ${ }^{1}$ Since major depression is approximately 20 times more common than schizophrenia, it can be seen that the per patient treatment costs are much higher in schizophrenia. In a more recent study, Knapp ${ }^{2}$ found that the annual identifiable direct and indirect costs (i.e. productivity losses) of schizophrenia in England were $£ 2.6$ billion, but even this sum omitted a number of indirect impacts.

\section{Need for Economic Evaluation of New Treatments}

Recent changes in the structure and funding of health services in the UK and elsewhere have focused attention on the increasing costs of healthcare and the need for costeffective treatments. In order to provide a cost-effective 
service, the components of 'cost' and 'efficacy' are being increasingly closely scrutinized.

Drugs for schizophrenia have not escaped this scrutiny, especially now that several new agents are available, with acquisition costs substantially higher than established therapies. The first of these drugs to raise economic concerns was clozapine. Being heralded as the first pharmacological breakthrough in the treatment of schizophrenia for 20 years, it was likely to attract a considerable amount of clinical interest, and hence usage. The same is true of other new drugs, such as risperidone and olanzapine, and a number of economic evaluations have been published, or are in progress. Since the new agents have a price much higher than that of the conventional neuroleptic drugs, and may also give rise to a temporary increase in rehabilitation costs, the obvious question is whether they generate additional benefits to justify the extra costs. For example, if the new agents have fewer side effects or encourage better compliance, this may result in improvements in outcomes or reductions in other costs, which may offset the higher acquisition costs of the drugs (see Figure 1).

\section{Existing Studies and Their Weaknesses}

The first economic evaluation involving the new drugs for schizophrenia was carried out in the USA by Revicki et $a l{ }^{3}$ on clozapine. They compared the costs and clinical outcomes of care over a two-year period for 133 patients started on clozapine with 51 patients treated with conventional neuroleptics. This economic study was not based on a prospective randomized trial, but attempts were made to match the two groups of patients.

In the year before the study began, the costs of care for the group subsequently given clozapine were higher than those of the group that continued on conventional neuroleptic therapy. This difference was maintained in the first year of the study but, by the end of the second year, the average costs of mental health services for the clozapine group (excluding the cost of clozapine) had fallen below those of the group continuing on conventional neuroleptic therapy, primarily as a result of a reduction in hospital costs. The authors concluded that, after two years, the net savings to the mental health system may be between $\$ 12000$ and $\$ 15000$ per patient.

A number of limitations and qualifications to this study have been noted, by both the authors and other commentators. The main problem relates to the historical cohort design. The clozapine patient group was itself highly selected and, despite attempts to find a matching group of patients receiving conventional neuroleptic care, the two groups differed in several demographic characteristics and with respect to their Brief Psychiatric Rating Scale (BPRS) scores. Also, different methods of measurement were used for the two groups of patients. The clozapine patients were participating in an ongoing clinical study in which data on the use of aftercare services were easily available, resulting in a more accurate enumeration of these services for clozapine patients than for patients treated with conventional neuroleptics.

Not all of the limitations would necessarily bias the study in favour of clozapine. However, a major concern was that the study excluded consideration of the costs and outcomes for those patients (around 35\%) who discontinued clozapine therapy, although the reasons for discontinuation were given. In a reworking of the analysis including drop-outs, Revicki et al. concluded that the net savings to the mental health system could be considerably lower, around \$900-7500 per patient. ${ }^{4}$

A second study, performed in the UK, by Davies and Drummond, ${ }^{5}$ adopted a modelling approach, using data from the earlier US study, ${ }^{3}$ supplemented by data from other sources. Given differences in clinical practice and organization of psychiatric services between the US and the UK, the results of the earlier US study were not readily applicable to the UK. (For example, rates of hospitalization for schizophrenia were known to differ between the two settings.)

The method employed was to construct a clinical decision tree. ${ }^{6}$ Data for some of the probabilities in the tree were taken from the US study (e.g., the probability of clozapine being discontinued, or the probability of clozapine patients attaining a particular BPRS score). However, other data, relating to the effectiveness of conventional neuroleptic

\section{Treatment Intermediate Outcomes \\ Costs and Cost Costs Reductions in longer term from:}
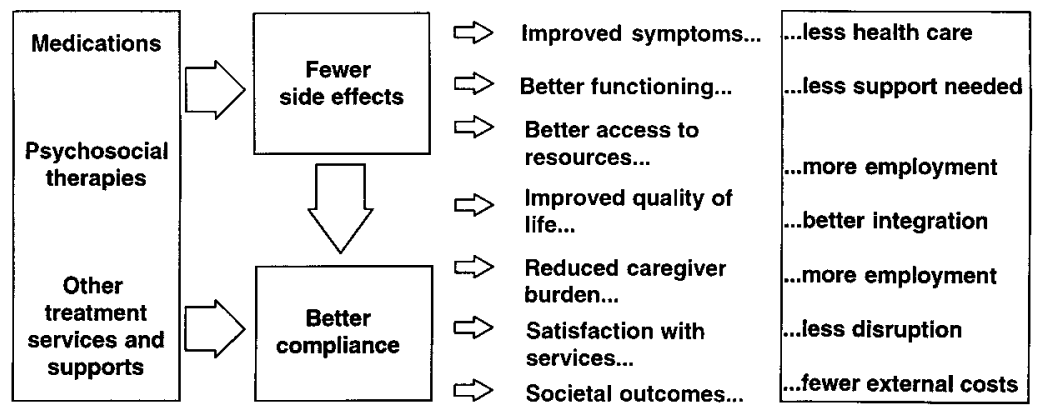

Figure 1. Hypothesized relationship between treatment costs, patient outcomes and economic benefits in schizophrenia 
therapy or the type of community based care received by patients discharged in either group, were used to supplement those from the US study. In particular, a Delphi (consensus) panel of five UK psychiatrists was used to assess the level of resource use in the UK setting for patients of different clinical status.

Davies and Drummond ${ }^{5}$ concluded that clozapine would lead to a net gain of 5.87 years of life 'with no disability or only mild disability' (defined as a BPRS score $<35$ or Clinical Global Impression Scale score $<3$ ). Also, the direct costs of using clozapine (including the costs of the drug) would be $£ 91$ less per annum, or $£ 1333$ per lifetime less than that for conventional neuroleptic therapy.

In a third study, Meltzer et al. ${ }^{7}$ collected data on 96 treatment-resistant patients with schizophrenia for two years before they entered a clozapine treatment study and for at least two years after. Information was obtained on the cost of inpatient and outpatient treatment, housing costs, other costs and family burden through direct interview or questionnaire.

It was found that the costs of treatment significantly decreased in the patients who continued clozapine treatment for at least two years. This was primarily due to a dramatic decrease in the frequency and cost of hospitalization. Costs were not, however, significantly lower in patients who dropped out of treatment. Taking into account all patients, there was a saving of $\$ 8700$ per patient per year over the two years, but there was a decrease in total costs of \$22936 per year for the 37 patients who continued clozapine and for whom cost data were available. Clozapine also produced a marked improvement in BPRS total scores as well as positive and negative symptoms scores, Global Assessment Scale scores, Quality of Life Scale scores, work functioning, capacity for independent living and rehospitalization rates.

Whereas this study represents an improvement over the studies discussed above, the before-and-after design suffers from the weakness that other factors, changing over the time period studied, could have had an impact on hospitalization (e.g., general budgetary pressures leading to a rationalization of bed capacity, or a growing reliance on community care).

Another new antipsychotic drug to be subjected to economic assessment is risperidone. Addington et al. ${ }^{8}$ retrospectively analysed hospitalization records for 74 patients enrolled in a one year open-label extension to a clinical trial. The number of hospital days were recorded for the 27 patients (36\%) who completed 365 days of openlabel therapy and compared with the number of days in the preceding 365-day period. It was found that the number of hospital days was reduced by $20 \%$ in those patients who responded to the drug.

This study has a number of weaknesses. First, only hospital days were considered. Whilst this is a major component of resource use, the consumption of other

aThe commercial name of quetiapine is Seroquel®, a trademark of Zeneca Pharmaceuticals.

bESTO: Evaluation of Seroquel on Treatment Outcomes. resources in the community could have changed. Secondly, only those patients who responded to the drug were included. Therefore, the study cannot give an overall picture of the cost implications of using risperidone.

A second study of the economic implications of risperidone was undertaken by Guest et al. ${ }^{9}$ They considered the resource use, gathered retrospectively, of 31 patients enrolled in the Swedish portion of a clinical trial, comparing utilization during the two years of risperidone treatment with that for the year before treatment. Costs from the UK were applied to the Swedish resource use data. Total mean cost (including hospital and residential accommodation costs) fell over the two years after commencing treatment with risperidone. The authors concluded that switching patients from conventional neuroleptics to risperidone improved the clinical outcome for some patients and potentially reduced costs for both the NHS and society as a whole. Obviously this study has many of the weaknesses of the other studies discussed above.

\section{Need for Prospective, Randomized Studies}

Therefore, it can be seen that the existing economic evaluations of drugs for schizophrenia have many weaknesses. The 'before and after' studies run the risk that other changes in health care over the period studied could impact on factors such as hospitalization. Also, some of the studies concentrate only on the responders to drug therapy and modelling studies are heavily reliant on assumptions made by the analyst, some of which may be hard to justify.

Prospective, randomized studies offer the best chance of minimizing bias and measurement error. Economic evaluations based on adequate and well controlled clinical studies are also favoured by the FDA. ${ }^{10}$ However, such studies raise a number of methodological challenges ${ }^{11}$ and there are still very few examples in the field of mental health.

The aim of this paper is to discuss how the key design issues were resolved in the planning of a prospective, randomized study to assess the socio-economic impact of a new atypical antipsychotic (quetiapine) ${ }^{\mathrm{a}}$. The study used as our example is the ESTO ${ }^{\mathrm{b}}$ study, an international multicentre randomized controlled trial, with concurrent data collection for a wide range of clinical, economic and quality of life outcomes. The study formed part of the phase III clinical programme for the drug concerned, but was designed with the explicit purpose of exploring its broader social and economic impact.

\section{Key Issues In Study Design}

\section{Patient Recruitment and Entry Criteria}

Much previous research has focused on chronic hospitalized patients and the need to discharge them from hospital. Today relatively few patients are long-term inpatients. Therefore, the challenge in the ESTO study was to find inclusion criteria that would define a group of representative 
patients, containing the sub-set of individuals still suffering exacerbations on their current drug therapy.

This proved difficult, as an 'acute exacerbation' does not necessarily lead to hospitalization. Also, since acute exacerbations, however defined, may not occur very frequently, what else should be taken as a marker of problems with current medication?

In the ESTO study a patient was considered to have an acute exacerbation 'where a stable or relatively stable patient, who may still have some symptoms, experiences an emergence of new symptoms or an exacerbation of existing symptoms, such that the patient's behaviour or social function is adversely affected'.

Patients were enrolled into the study if, in addition to the presenting exacerbation, they had had at least one hospitalization or documented evidence of exacerbation within the previous three years. Also, it was originally stated that patients must have been diagnosed with schizophrenia within the last five years. However, this condition was subsequently dropped in order to aid recruitment to the study.

\section{Selection of Comparator Drug}

The official guidelines for economic evaluation of pharmaceuticals state that the most appropriate comparator drug to a new agent is 'the drug most likely to be replaced', ${ }^{12}$ or 'existing practice'. ${ }^{13}$ However, in reality 'existing practice' can vary from place to place or change over time. Many clinical trials of new pharmaceuticals are conducted in a number of centres, often from more than one country, so the choice of comparator drug is often a compromise.

It was recognized that there were international differences in drug therapy for schizophrenics and also that, after many years with no new agents, some were now being launched. As it was known that the ESTO study would not report for two years, a judgement had to be made about whether any of the new agents (e.g., risperidone) would become sufficiently established in the intervening period to be regarded as 'current practice'. It was decided that, despite the growing popularity of the newer agents, conventional neuroleptics would still be the most relevant comparators.

Haloperidol was identified as the most widely used neuroleptic medication in the countries studied. In a similar study, being undertaken in the United Kingdom only, chlorpromazine was chosen as the comparator because of its greater use. ${ }^{14}$ On admission to the ESTO study, existing psychotic medication was withdrawn, prior to randomization to quetiapine or haloperidol, although benzodiazapines and anticholinergics can be given to patients with acute agitation. Doses of both study drugs were titrated up to an optimal dose, with flexibility for additional increases if required, as would be the case in normal clinical practice (see Figure 2).

\section{Blinding of Doctor and Patient}

Economic evaluation seeks to mirror what would happen in real life. Therefore, the ideal clinical trial for concurrent economic analysis would be one that has an unobtrusive protocol, which requires neither the patient nor the doctor to behave any differently than they would in normal clinical practice. ${ }^{11}$ This is clearly at odds with blinding of doctor and patient to therapy, since this often requires patients to take additional dummy medications and effectively rules out the use of depot injections, since these are not normally available for new agents. On the other hand, it is possible that failure to blind such a study could introduce biases, since it would be clear to both doctor and patients that some subjects were being given a new medication. Therefore, prior beliefs about the superiority of a new compound may influence perceptions of its benefit. ${ }^{15}$

Both patients and doctors were blinded to treatment allocation in the ESTO study, a decision taken on the grounds that, since quetiapine is still in development, unblinded assessments of efficacy would not be credible. This being the case, the study may run the risk of poor compliance because of the need for the patients to take additional, dummy medications resulting in a complicated treatment regimen. Also, since depot injections were not available under the protocol, patients may withdraw from study medication if their doctor considers that depots are required. However, they continue to be followed up for the full period of the study.

\section{Range of Socio-Economic Outcomes}

Schizophrenia has broad socio-economic impacts. Therefore, any economic evaluation of therapy must include consideration of a wide range of costs and consequences. The starting point for the discussion was the range of outcomes outlined in Figure 1.

\section{Direct Costs}

For an economic evaluation the most obvious outcomes are the direct costs, which fall predominantly, though not exclusively, on the healthcare sector. For diseases such as schizophrenia many costs are likely to fall in the community care sector, or on the family itself. Typically, these are more difficult to track than (say) hospital costs, so consideration needs to be given as to whether they are sufficiently important to justify the extra time and effort required by their measurement.

Also, families often bear a considerable burden associated with the care of a relative with schizophrenia and may incur direct costs. This may be particularly the case, given the increasing emphasis being placed on communitybased treatment.

\section{Productivity Changes}

The more seriously ill patients have, at present, little prospect of a return to regular employment, but moderately ill patients may be able to obtain a job, given improvements in clinical and social functioning. The inclusion of productivity changes in economic evaluations in health care is controversial, ${ }^{16,17}$ but consideration was given to their inclusion in the ESTO study. 


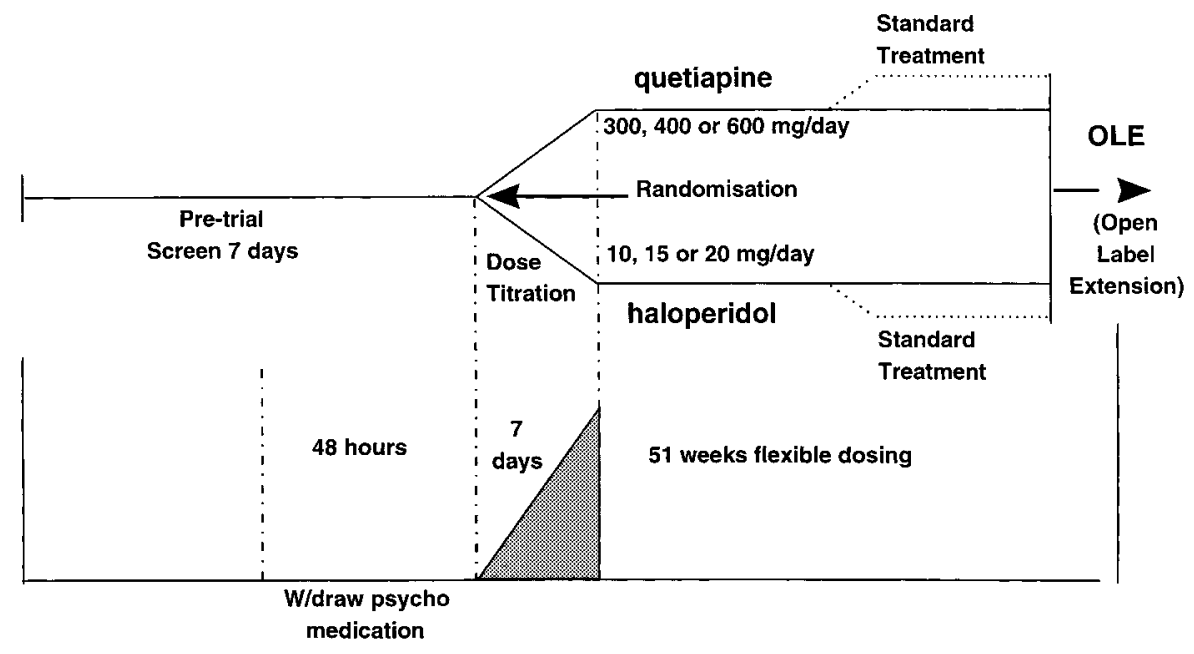

Figure 2. Design: multicentre, double-blind, randomized, comparison of treatment strategies in patients initially treated with 'quetiapine' and haloperidol

\section{Quality of Life}

Another important outcome is quality of life, which can be affected by both the efficacy of the drugs given and their side effects. In addition, the burden placed on caregivers could reduce their quality of life. There are three types of quality of life scale: (disease) specific measures, general health profiles and preference-based measures. Each has its particular attributes and uses. Specific measures, being focused on the disease in question, are the most likely to show a change if the patient's condition improves or worsens. General health profiles (such as the SF-36) ${ }^{18}$ enable the quality of life of schizophrenia patients to be compared with that of individuals suffering from other diseases. Preference-based measures (such as the EuroQoL/EQ5D) ${ }^{19}$ would enable the quality-adjusted life years (QALYs) gained to be calculated and hence a cost-utility analysis to be performed. ${ }^{17}$

All three categories of measure were considered in the design of the ESTO study, bearing in mind the constraint that inclusion of too many data collection instruments would overburden investigators and their patients. Other factors considered in the choice of instruments were whether they were available in the languages required and whether they were self-administered or required an interviewer.

Finally, although the main focus of the ESTO study was to detect changes in the socio-economic outcomes identified in Figure 1, consideration was also given as to whether it was necessary to retain a number of the traditional clinical measures used in schizophrenia studies. Whilst not directly useful for the economic analysis, they can provide empirical support for the criterion validity of the other changes observed. For example, it is important to ascertain whether changes in resource use and quality of life are, in fact, accompanied by changes in psychopathology and/or sideeffects. Therefore, it is particularly important to include a number of such measurements in all clinical trials of a new drug prior to licensing.

\section{Measurements Included}

A number of clinical endpoints were included as outlined in Table 1. The primary purpose was to assess whether changes in socio-economic endpoints were indeed matched by changes in the patient's clinical condition, thereby adding some validity to any economic claims for the new drug. Therefore, the Positive and Negative Syndrome Scale (PANSS) $^{20}$ was included, as was the Clinical Global Impressions (CGI), the former being primarily based on a 30-40 min patient interview, the later being an evaluation made by the patient's physician. In addition, the $\mathrm{AIMS}^{21}$ neurological rating scale was used to measure dyskinetic movements and the neurological rating scale of Simpson and Angus $^{22}$ was used for the evaluation of extrapyramidal symptoms (EPS).

It was also decided to track a wide range of socioeconomic measures. (The schedule of trial measurements is given in Table 1.) Direct health service costs (e.g., hospital days, outpatient visits, medications) and other aspects of resource use in the community were assessed through the Client Service Receipt Inventory (CSRI). ${ }^{23}$ This is a flexible instrument designed to collect information on service receipt, service-related issues and income. It enables the costs of care packages to be calculated. ${ }^{24}$ The main sections of the instrument cover background and client information, accommodation, employment, earnings, other personal resources and service receipt. Prior to its use in the ESTO study, the CSRI was modified after discussions with analysts in the countries concerned, in order to ensure that all the terms were understood.

It was decided to assess patient quality of life by the Short-Form $36,{ }^{18}$ a widely used and validated general health profile measure. It was chosen because it was available in validated translation for all the countries where the trial was being run and because it would allow comparisons of disease states in schizophrenia with those of other diseases. The use of a (disease) specific measure was considered and then 
Table 1. Schedule of trial measurements

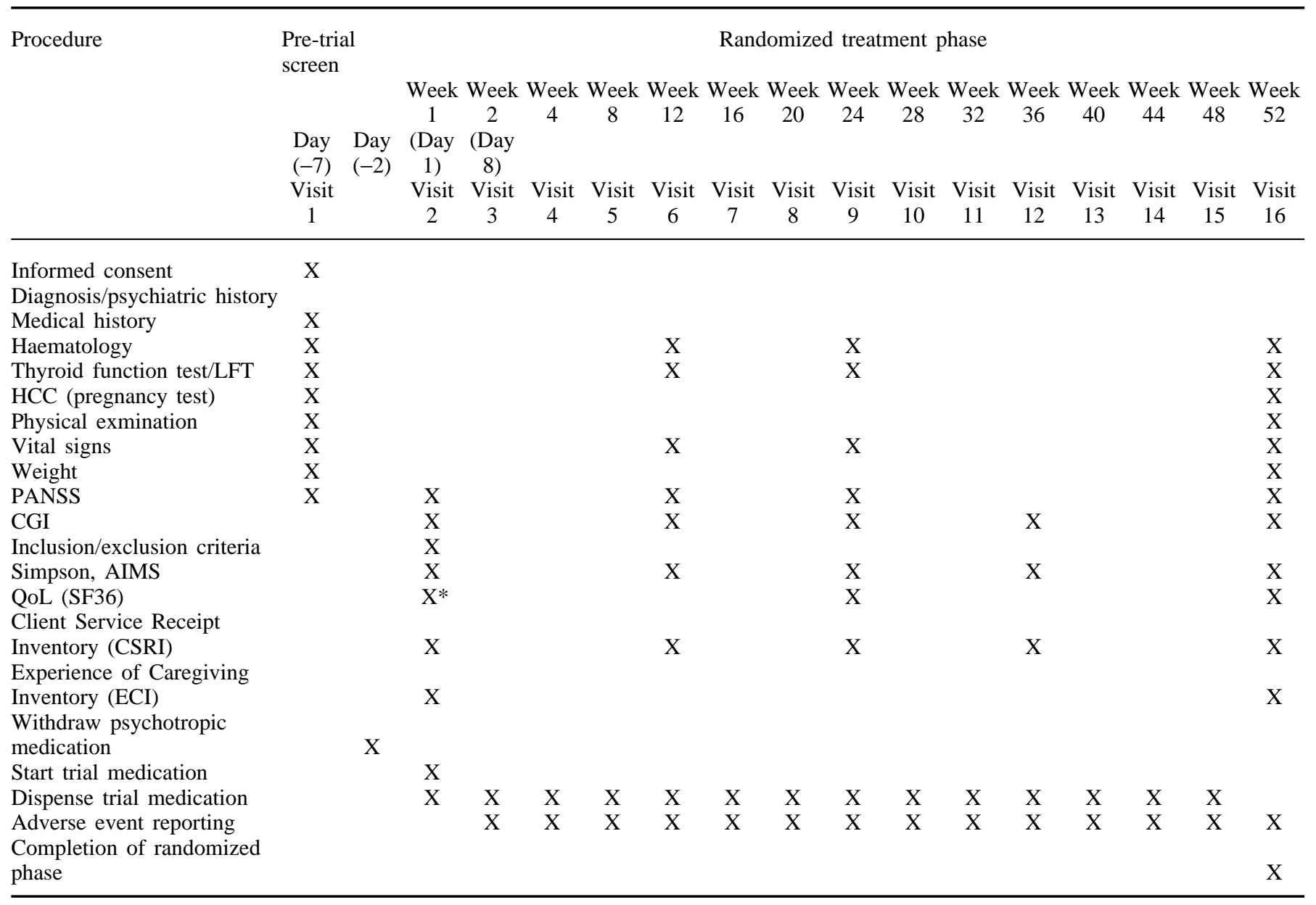

* Or within $14 \mathrm{~d}$.

rejected on the grounds that none had been translated and validated in all the languages required. However, a disease specific measure, the Lancashire Quality of Life Profile, ${ }^{25}$ is being used in a similar trial being conducted in the United Kingdom only. ${ }^{14}$

Caregivers' experiences are being assessed through the Experience of Caregiving Inventory (ECI). ${ }^{26}$ This is a selfreport measure of the experience of caring for a relative with serious mental illness, conceptualized within a 'stressappraisal-coping' framework. By rejecting the notion of 'burden', the ECI avoids the problematic distinction between 'objective' and 'subjective' burden and allows both the positive and negative aspects of caregiving to be examined.

\section{Length of Follow-Up}

Many clinical trials in schizophrenia are of short duration (e.g., 6-8 weeks). A major reason for this is that schizophrenia patients are often poor compliers; therefore, a longer trial may risk having many drop-outs. Also, currently a number of new drugs and new forms of psychosocial intervention for schizophrenia are being developed. Therefore, clinical centres may be asked to participate in trials of many new agents and thus a long-term trial with any particular agent may use up a high proportion of the total available caseload for clinical research. Moreover, pharmaceutical companies wish to reduce the time required for clinical research to the minimum.

However, short-term trials pose problems for economic analysis, as many of the changes in the socio-economic outcomes may take some time to occur. This can be seen in the earlier, uncontrolled, economic studies reviewed above, where reductions in hospital costs did not become apparent until at least one year.

Therefore, consideration was given as to whether a longterm study would be advisable and, if so, the likely consequences in terms of drop-outs and the ways of dealing with such a problem.

It was decided to follow patients for 1 year, irrespective of whether they remained on study medication. On withdrawal from study medication the physician is free to use the agent of his or her choice, with the exception that quetiapine is not allowed, being an investigational medicine. Therefore, the question being asked is 'What are the clinical, cost and quality of life outcomes over 1 year for those patients initially treated with quetiapine or haloperidol?' Clearly the extent to which the study will tell us about the difference between the efficacy of the two 
study drugs will depend on the proportion of withdrawals in either group.

\section{Sample Size}

Given the primary focus on socio-economic outcomes, the ideal sample size calculation for such a study would be one based on the ability to detect a given change in a primary economic endpoint. However, very little is known about the distribution of key economic variables, such as total cost or cost-effectiveness, for this group of patients. Also, some of the statistical issues in testing for differences in costeffectiveness ratios have not been resolved and the size of the 'economically important difference' is not easily defined. ${ }^{27}$ As a consequence, many economic evaluations conducted alongside clinical trials in psychiatry have been underpowered. ${ }^{28}$

The sample size calculation was based on showing a difference between quetiapine and haloperidol in the proportion of patients who discontinued randomized treatment. This approach was followed because it was not possible to identify a suitable primary economic endpoint on which to power the study. Nevertheless, it was thought that discontinuation of treatment would be highly correlated with additional costs and deterioration in quality of life.

No information was available on the rate of withdrawal from haloperidol over a 1 year period, so conservative estimates were used. If $60 \%$ of patients randomized to haloperidol discontinued randomized treatment, and the corresponding proportion for quetiapine was at least $15 \%$ lower (i.e., $45 \%$ or less), 190 patients per group would be required, with alpha at the $5 \%$ level and $80 \%$ power.

The analysis will be conducted using logistic regression techniques, including the randomized treatment centre, and centre-by-treatment interaction as factors. Odds ratios will be estimated to compare the treatments and $95 \%$ confidence intervals calculated. The ratios will be compared at the $5 \%$ level of significance using a two-sided test.

\section{Conclusions}

The design of studies such as ESTO is inevitably a compromise between control and pragmatism. For example, whilst blinding may reduce potential bias, this may cause difficulty with compliance. Despite these compromises, the ESTO study should provide a more reliable assessment of the socio-economic outcomes of a new anti-psychotic and has attracted the widespread support of analysts and investigators. It has already served as a template for other studies $^{14}$ and, if the methodology is successful, will have implications for the assessment of similar drugs in the future.

\section{Acknowledgements}

Helpful comments and advice have been received from a number of individuals. We are particularly grateful to Jenny Beecham, Jim Brown, Nick Freemantle, Bengt Jonsson, Sue
Langham and Richard Williams. We also acknowledge helpful comments from the anonymous referees. However, we are solely responsible for the views expressed. Earlier versions of the paper were presented as a poster at the ENCP meeting in Amsterdam, 21-25 September 1996, and at an ARCAP symposium on the economics of mental health in Venice, 14-16 March 1997.

\section{References}

1. Davies LM, Drummond MF. Economics and schizophrenia: the real cost. Br. J. Psychiatry 1994; 165 (suppl. 25): 18-21.

2. Knapp MRJ. Costs of schizophrenia. Br. J. Psychiat 1997; 171 (6) 509-518.

3. Revicki DA, Luce BR, Weschler JM, Brown RE, Adler MA. Costeffectiveness of clozapine for treatment-resistasnt schizophrenics. Hospital Community Psychiatry 1990; 41: 850-854.

4. Revicki DA, Luce BR, Weschler JM, Brown RA, Adler MA Clozapine's cost-benefits. Reply. Hospital Community Psychiatry 1991; 42 (1): 93-94.

5. Davies LM, Drummond MF. Assessment of costs and benefits of drug therapy for treatment-resistant schizophrenia in the United Kingdom. Br. J. Psychiatry 1993; 162: 38-42.

6. Weinstein MC, Fineberg HV. Clinical Decision Analysis. Philadelphia, PA. Saunders, 1980.

7. Meltzer HY, Cola P, Lynne W, Thompson PA, Bastani B, Davies MA, Snitz B. Cost-effectiveness of clozapine in neuroleptic-resistant schizophrenia. Am. J. Psychiatry 1993; 150: 1630-1638.

8. Addington DE, Jones B, Bloom D, Chouinard G, Remington G, Allbright P. Reduction of hospital days in chronic schizophrenic patients treated with risperidone: a retrospective study. Clin. Therapeut. 1993; 15 (5): 917-926.

9. Guest JF, Hart WM, Cookson RF, Lindstrom MD. Pharmacoeconomic evaluation of long-term treatment with risperidone for patients with chronic schizophrenia. Br. J. Med. Econ. 1996; 10: 59-67.

10. Food and Drug Administration (FDA). Draft guidelines for pharmacoeconomic claims. Rockville, MD: FDA.

11. Drummond MF, Davies LM. Economic analysis alongside clinical trials: revisiting the methodological issues. Int. J. Technol. Assessment Health Care 1991; 7 (4): 561-573.

12. Commonwealth of Australia. Guidelines for the pharmaceutical industry on preparation of submissions to the Pharmaceutical Benefits Advisory Committee (PBAC): including major submissions involving economic analysis. Canberra: Department of Human Services and Health, 1995.

13. Canadian Coordinating Office for Health Technology Assessment (CCOHTA). Guidelines for economic evaluation of pharmaceuticals: Canada, 1st edn. Ottawa: CCOHTA, 1994.

14. Zeneca Pharmaceuticals. A multicentre, double-blind, randomised tria to compare the effects of Seroquel and Chlorpromazine on treatment outcomes for schizophrenic patients. Trial 5077UK/001 SOS Wilmslow: Zeneca, 1996.

15. Freemantle, N, Drummond MF. Should clinical trials with concurrent economic analyses be blinded? J. Amer. Medical Assoc. 1996; 277 (1): 63-64.

16. Gold MR, Seigel JE, Weinstein MC, Russell LB. Cost-effectiveness in health and medicine. New York: Oxford University Press, 1996.

17. Drummond MF, O'Brien BJ, Stoddart GL, Torrance GW. Methods for the economic evaluation of health care programmes, 2nd edn. Oxford: Oxford Medical, 1997.

18. Ware JE, Sherbourne CD. The MOS 36-item Short Form Health Survey (SF-36). I: conceptual framework and item selection. Med. Care 1992; 30: 247-283.

19. Kind P. The EuroQoL Instrument: an index of health-related quality of life. In: Spilker B, ed. Quality of life and pharmacoeconomics in clinical trials. Philadelphia, PA: Lippincott-Raven, 1996, 191-201.

20. Kay SR, Fizbein A, Opler LA. The positive and negative syndrome scale (PANSS) for schizophrenia. Schizophren. Bull. 1987; 13: 261-276.

21. Guy W. ECDEU assessment manual for psychopharmacology, revised edn. Washington, DC: US Department of Health Education and Welfare, 1976. 
22. Simpson GN, Angus JWS. A rating scale for extra pyramidal side effects. Acta Psychiatr. Scand. 1970; 212 (suppl. 44): 11-19.

23. Beecham JK. Collecting information: the Client Service Receipt Interview. Mental Health Res. Rev. 1994; 1: 6-8.

24. Beecham, JK, Knapp MRJ. Costing psychiatric interventions. In Thornicroft G, Brewin, C, Wing JK, eds. Measuring mental health needs. London: Gaskell, 1992, 163-183.

25. Olwer JPJ. The social care directive: development of a quality of life profile for use in community services for the mentally ill. Social Work Services Rev. 1991; 3: 5-45.

26. Szmukler GI, Burgess P, Herrman H, Benson A, Colusa S, Bloch S.
Caring for relatives with serious mental illness: the development of the Experience of Caregiving Inventory. Social Psychiatry Psychiatric Epidemiol. 1993; 31 (3-4): 137-148.

27. Drummond MF, O'Brien BJ. Clinical importance, statistical significance and the assessment of economic and quality-of-life outcomes. Health Econ. 1993; 2 (3), 205-212.

28. Gray A, Marshall M, Lockwood A, Morris J. Problems in conducting economic evaluations alongside clinical trials: lessons from a study of case management of people with mental disorders. Br. J. Psychiatry 1997; 170: 47-52. 